DESY-16-225

\title{
Parton Distributions from Lattice QCD with Momentum Smearing
}

\section{Constantia Alexandrou ${ }^{a b}$, Krzysztof Cichy $^{c d}$, Martha Constantinou ${ }^{e}$, Kyriakos Hadjiyiannakou $^{a}$, Karl Jansen $^{f}$, Fernanda Steffens $^{f}$, Christian Wiese $^{* f}$}

${ }^{a}$ Department of Physics, University of Cyprus, P.O. Box 20537, 1678 Nicosia, Cyprus

${ }^{b}$ Computation-based Science and Technology Research Center, Cyprus Institute, 20 Kavafi Str., Nicosia 2121, Cyprus

${ }^{c}$ Goethe-Universität Frankfurt am Main, Institut für Theoretische Physik, Max-von-Laue-Strasse 1, 60438 Frankfurt am Main, Germany

${ }^{d}$ Faculty of Physics, Adam Mickiewicz University, Umultowska 85, 61-614 Poznań, Poland

${ }^{e}$ Temple University, 1925 N. 12th Street, Philadelphia, PA 19122, USA

${ }^{f}$ John von Neumann Institute for Computing (NIC), DESY, Platanenallee 6, D-15738 Zeuthen, Germany

E-mail: christian.wieseddesy.de

In this work we continue our effort to explore a recent proposal, which allows light-cone distributions to be extracted from purely spatial correlations, being thus accessible to lattice methods. In order to test the feasibility of this method, we present our latest results from a twisted mass lattice calculation of the flavor non-singlet momentum, helicity and transversity distributions of the nucleon. Furthermore, we apply a newly proposed momentum improved smearing, which has the potential to reach higher nucleon momenta as required for a safe matching procedure to the physical distribution functions.

34th annual International Symposium on Lattice Field Theory

24-30 July 2016

University of Southampton, UK

\footnotetext{
* Speaker.
} 


\section{Introduction}

Although parton distribution functions are the fundamental objects describing the inner structure of hadrons, they were so far not calculated from first principles. In the past, lattice QCD has successfully been employed for the computation of hadronic spectra and form factors, for instance. Yet calculations of quark distributions are still missing, since they are given by light-cone correlation functions and light-like distances are not accessible on an Euclidean lattice.

A new method to deal with this problem was proposed in Ref. [1] and employs the computation of a purely spatial quasi-distribution in a finite momentum frame. How to relate this quasidistribution to the physical PDF and general studies of this method have already been addressed in a handful of papers, e.g. [2, 3, 4, 5, 6, 7].

A crucial point when making the connection to PDFs is a large momentum limit for the nucleon boost. On the lattice, this provides a challenge, since large momentum nucleon observables are known to be very noisy. Thus, we study the effect of including a momentum dependent quark field smearing [8] into our calculation, in order to improve the quality of the signal, especially for large nucleon momenta.

A comprehensive work on the calculation of PDFs with more elaborate studies of the recent developments and our newest results can be found in our latest paper [9].

\section{Lattice calculation \& momentum smearing}

The quasi-distributions are computed from

$$
\tilde{q}\left(x, \Lambda, P_{3}\right)=\int_{-\infty}^{\infty} \frac{d z}{4 \pi} e^{-i z k_{3}}\left\langle P\left|\bar{\psi}(z) \gamma_{3} W_{3}(z, 0) \psi(0)\right| P\right\rangle,
$$

where $W_{j}(z, 0)$ is the Wilson line from 0 to $z$ in the spatial $j$ direction, $k_{3}=x P_{3}$ and the Euclidean momentum is $P=\left(0,0, P_{3}, P_{4}\right)$. It is required that the Wilson line and the spatial nucleon momentum boost point into the same direction. In lattice $\mathrm{QCD}$, one can compute matrix elements of operator as suitable ratios of three- and two-point correlation functions. The full set of PDFs can be accessed by using three-point functions where the inserted operators have the Dirac structures

- $\gamma_{3}$, for the case of the unpolarized momentum quasi-distributions $\tilde{q}\left(x, \Lambda, P_{3}\right)$;

- $\gamma_{3} \gamma_{5}$, for the case of the helicity quasi-distributions $\Delta \tilde{q}\left(x, \Lambda, P_{3}\right)$;

- $\gamma_{3} \gamma_{j}(j=1,2)$, for the case of the transversity quasi-distributions $\delta \tilde{q}\left(x, \Lambda, P_{3}\right)$.

Due to the rotational invariance on the lattice, one is certainly not restricted to the 3-direction and can easily generalize the operators to the other two directions. In order to obtain a quasidistribution from the computed matrix elements a Fourier transformation has to be performed. The quasi-distribution can be related to the physical PDF by a one-loop matching and a mass correction. Details for these steps can be found in Refs. [4, 5], for example.

In former studies we applied standard Gaussian smearing to the nucleon fields in order to improve the overlap to the nucleon ground state. This, however, is only valid for momentum zero and deteriorates the signal for large momenta. Thus, in Ref. [8] a new type of smearing, the momentum 
smearing, is proposed. This smearing is constructed in a way to increase the overlap of the used nucleon field with a momentum boosted nucleon ground state. In contrast to the Gaussian smearing quark fields are smeared according to

$$
S_{\text {mom }} \psi(x)=\frac{1}{1+6 \alpha}\left(\psi(x)+\alpha \sum_{j} U_{j}(x) e^{i k \hat{j}} \psi(x+\hat{j})\right),
$$

where $k=\zeta P$, with $P$ the lattice momentum of the nucleon and $\zeta$ a tunable parameter. This form looks very similar to the definition of Gaussian smearing with an addition of an exponential factor multiplying the gauge links in the direction of the momentum boost. In practice, we use a Gaussian smearing routine with the standard nucleon parameters (50 steps, $\alpha=4)$ and include a gauge field with a complex phase $\tilde{U}_{j}(x)=U_{j}(x) e^{i k \hat{j}}$. For now we follow [8] and choose $\zeta$ to be 0.45 .

All calculations presented here were performed on an ETMC (European Twisted Mass Collaboration) gauge field ensemble [10], with $N_{f}=2+1+1$ flavors of maximally twisted mass fermions and a volume of $32^{3} \times 64$. The bare coupling is set to $\beta=1.95$, corresponding to a lattice spacing of $a \approx 0.082 \mathrm{fm}$. The twisted mass parameter is $a \mu=0.0055$, which gives a pion mass of $m_{P S} \approx 370 \mathrm{MeV}$. Because we still do not have a proper renormalization of the involved operator we apply 5 steps of HYP smearing [11] to the gauge links in the Wilson line. This is expected to bring the values of the renormalization constants close to their tree-level values.

For the computation of the matrix elements we used only 50 gauge configurations with 3 sequential propagators (one for each spatial direction), resulting in 150 measurements. In case of the unpolarized momentum distribution and $P_{3}=10 \pi / L$, we used 100 gauge configurations. Currently the source-sink separation is set to $t_{s}=8 a$, in order to get a better signal. Larger separations and thus the influence of excited states will be studied in the future.

\section{Results}

In order to show the quality of our lattice calculation, we show the unrenormalized matrix elements for the momentum, the helicity and transversity cases in Figs. 1, 2 and 3. To demonstrate the capabilities of the momentum smearing we show results for momenta up to $P_{3}=10 \pi / L$ for the unpolarized case. When comparing to previous results that employ the stochastic method [9] one can see that a factor of $\approx 200$ less measurements is necessary to obtain results with a comparable uncertainty for a single momentum.

Consequently, in Fig. 4 we show the momentum dependence of the unpolarized momentum quasi-distribution. One can see that for larger momenta there is a trend that points into the direction of the phenomenological PDF curves. However, in order to make proper statements here we will need probably even larger momenta and a procedure to extrapolate to very large or even infinite momentum. We show only the quasi-distributions in this plot, since the influence of the matching and mass correction is very small for momenta larger than $P_{3}=6 \pi / L$. This can especially be seen when looking at the results for the helicity and transversity distributions for $P_{3}=6 \pi / L$ in Fig. 5 . Here, the difference between the quasi-distribution, the finite mass distribution and the physical PDF is almost negligible. For even larger momenta there should be no visible difference. 

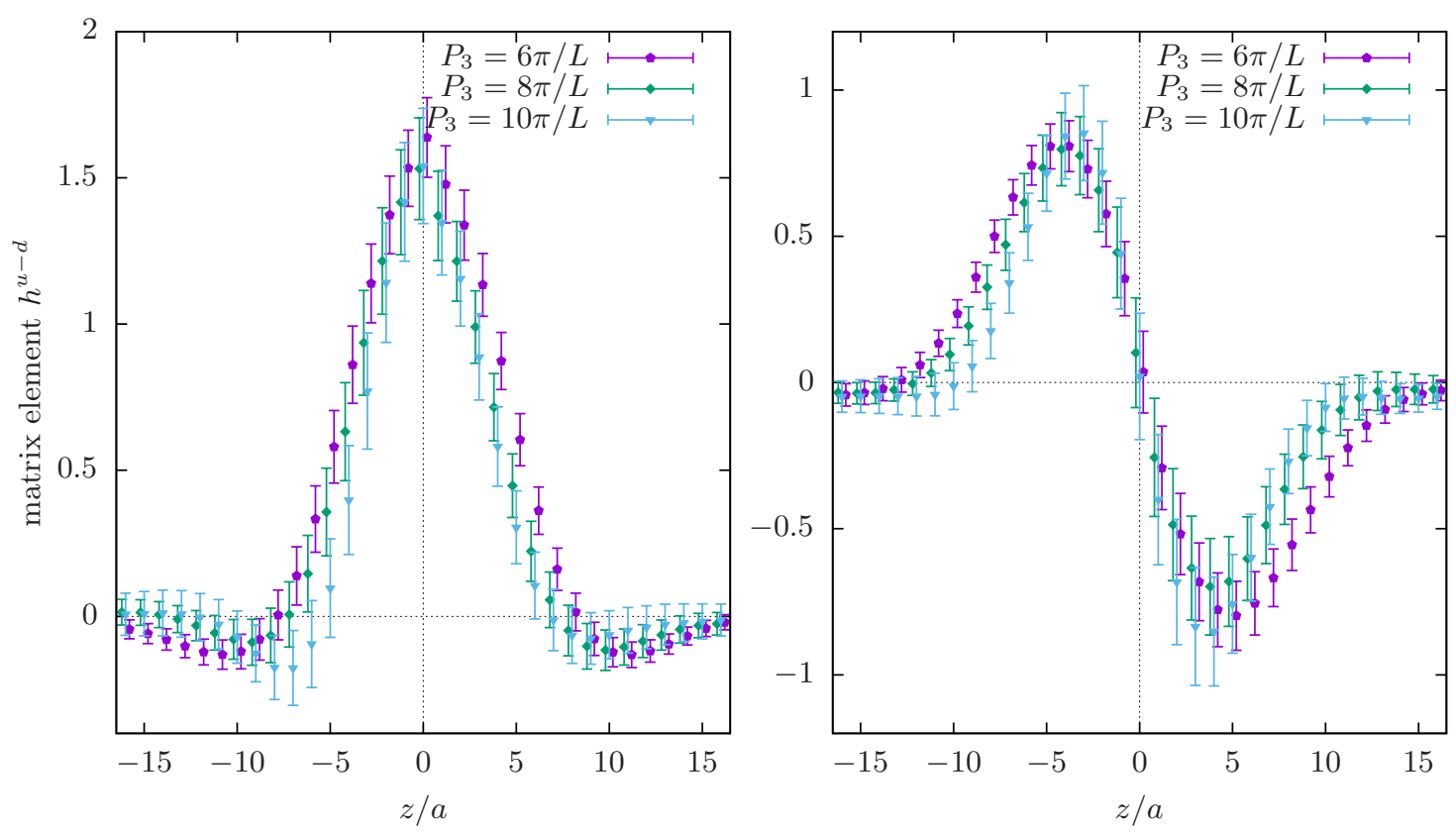

Figure 1: Unrenormalized matrix elements of the vector operator (for the momentum distribution) and three different nucleon boost momenta, left: real part, right: imaginary part.
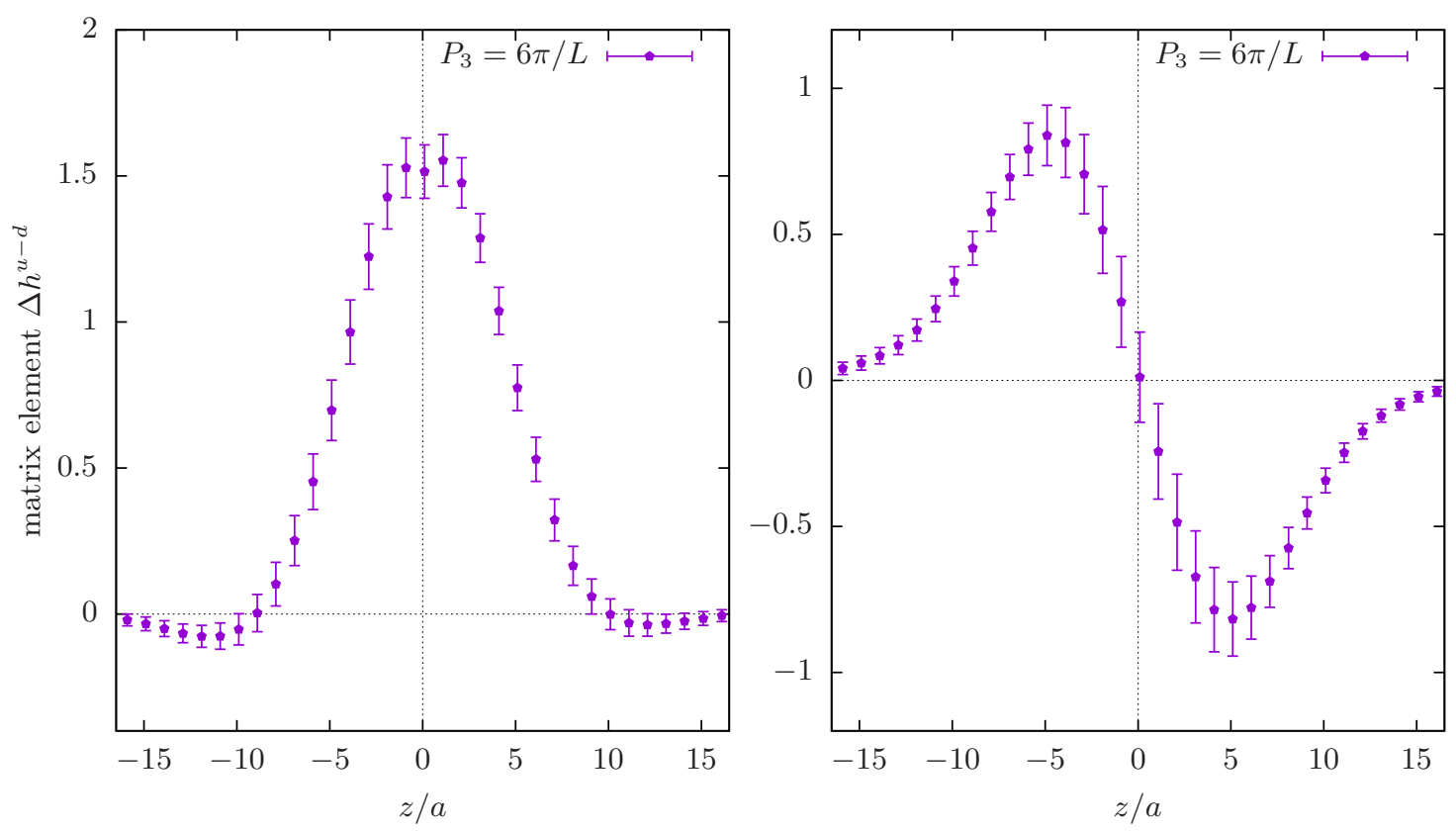

Figure 2: Unrenormalized matrix elements of the axial-vector operator (for the helicity distribution) and $P_{3}=6 \pi / L$, left: real part, right: imaginary part. 

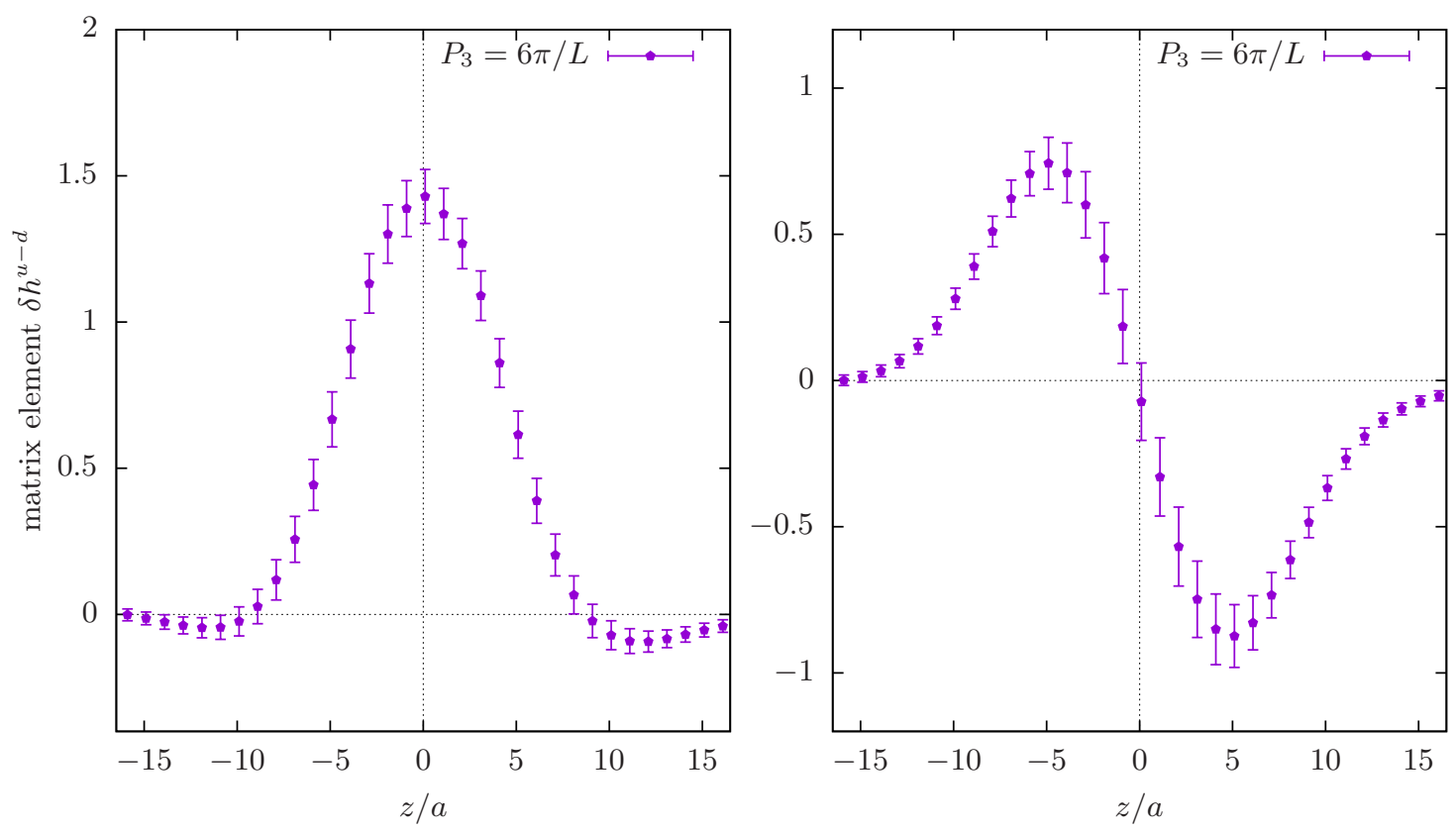

Figure 3: Unrenormalized matrix elements of the tensor operator (for the transversity distribution) and $P_{3}=6 \pi / L$, left: real part, right: imaginary part.

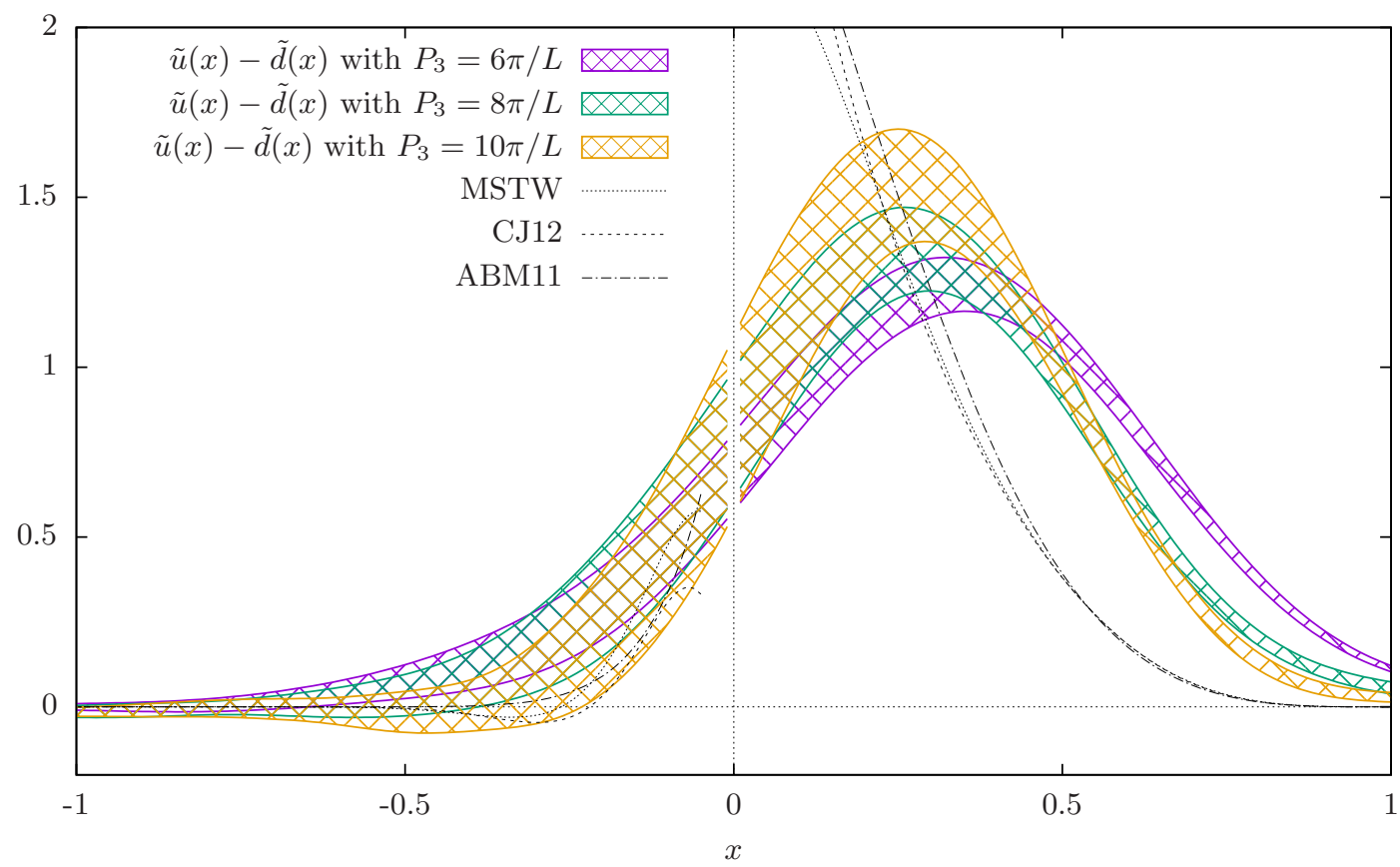

Figure 4: Momentum quasi-distribution for three different nucleon momenta. MSTW [12], CJ12 [13] and ABM11 [14] are phenomenologically extracted distributions plotted for orientation. 

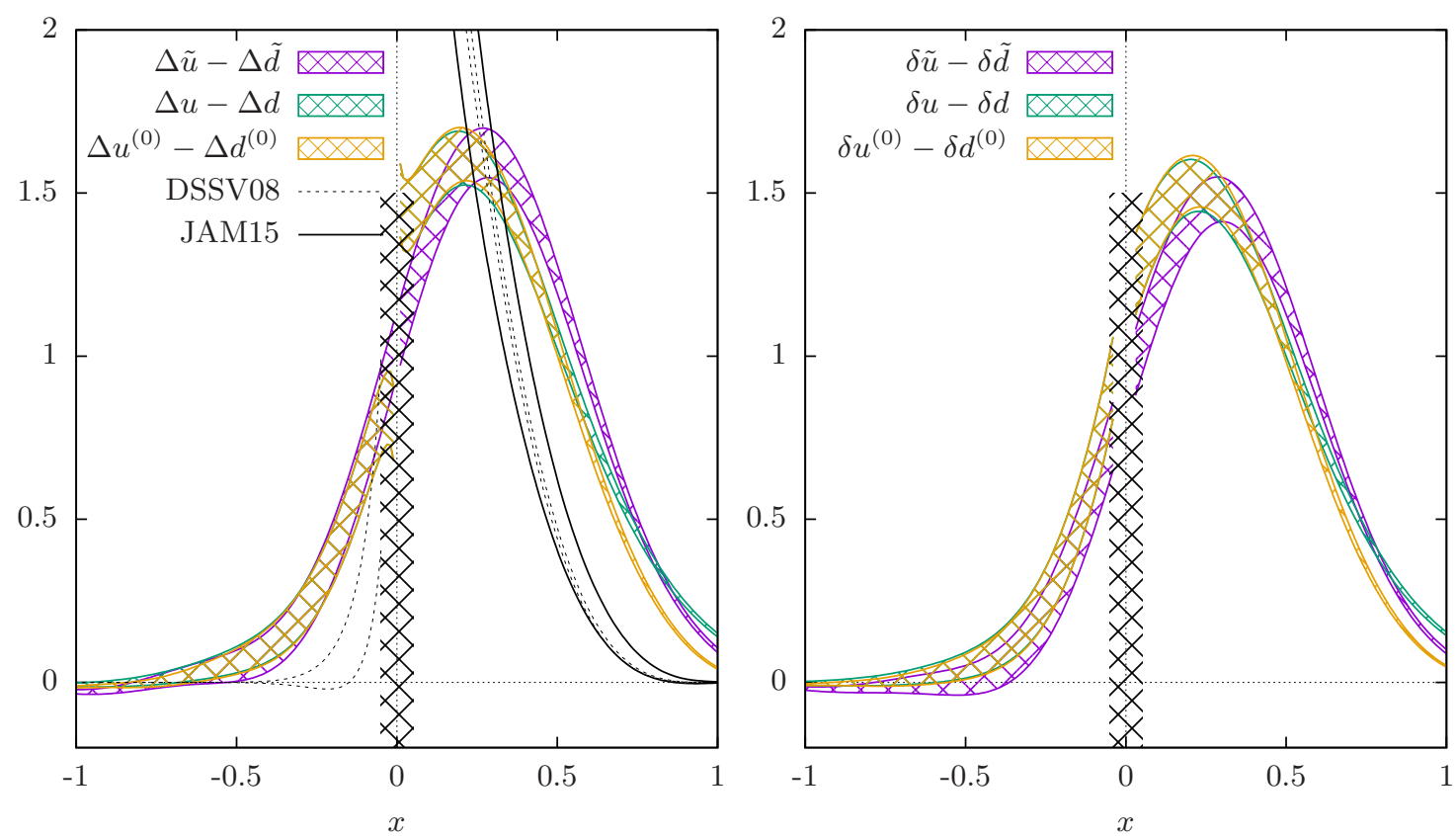

Figure 5: Quasi-distribution, finite nucleon mass distribution and PDF after nucleon mass corrections for $P_{3}=6 \pi / L$, left: helicity, right: transversity. DSSV08 [15] and JAM15 [16] are phenomenologically extracted distributions plotted for orientation.

\section{Conclusion and Outlook}

In this proceeding we gave a brief update on our current effort to compute parton distribution functions of the nucleon with lattice QCD methods. We especially focused on the recent inclusion of momentum dependent smearing. We were able to show that by using this smearing in combination with the sequential method it is possible to reduce the error for larger momenta up to a factor of 200 in comparison to our previous work where we used the stochastic method. We applied this new smearing to the momentum, the helicity and the transversity distribution.

In the future, the application of momentum smearing will thus enable us to reach sufficiently large momenta in order to make a connection to the physical light cone distribution. When other systematic effects, e.g. the quark masses and cut-off effects and, in particular, the question of renormalization, are under control, we will be able to tell, if PDFs can be extracted from a lattice QCD calculation.

\section{Acknowledgements}

K.C. was supported in part by the Deutsche Forschungsgemeinschaft (DFG), project nr. CI 236/1-1. F.S. was partly supported by CNPq contract number 249168/2013-8. We thank our fellow members of ETMC for their constant collaboration. In particular helpful discussions with G.C. Rossi are gratefully acknowledged. We are grateful to the John von Neumann Institute for Computing (NIC), the Jülich Supercomputing Center and the DESY Zeuthen Computing Center for their 
computing resources and support. This work has been supported by the Cyprus Research Promotion Foundation through the Project Cy-Tera (NEA YПО $\Delta \mathrm{OMH} / \Sigma T$ TPTH/0308/31) co-financed by the European Regional Development Fund. This work has received funding from the European Union's Horizon 2020 research and innovation programme under the Marie Sklodowska-Curie grant agreement No 642069 (HPC-LEAP).

\section{References}

[1] X. Ji, Parton Physics on a Euclidean Lattice, Phys.Rev.Lett. 110 (2013) 262002, [1305 . 1539 ].

[2] X. Xiong, X. Ji, J.-H. Zhang, and Y. Zhao, One-loop matching for parton distributions: Nonsinglet case, Phys.Rev. D90 (2014) 014051, [1310.7471].

[3] H.-W. Lin, J.-W. Chen, S. D. Cohen, and X. Ji, Flavor Structure of the Nucleon Sea from Lattice QCD, Phys. Rev. D91 (2015) 054510, [1402.1462].

[4] C. Alexandrou, K. Cichy, V. Drach, E. Garcia-Ramos, K. Hadjiyiannakou, K. Jansen, et al., Lattice calculation of parton distributions, Phys. Rev. D92 (2015) 014502, [1504.07455].

[5] J.-W. Chen, S. D. Cohen, X. Ji, H.-W. Lin, and J.-H. Zhang, Nucleon Helicity and Transversity Parton Distributions from Lattice QCD, Nucl. Phys. B911 (2016) 246-273, [1603. 06664 ].

[6] T. Ishikawa, Y.-Q. Ma, J.-W. Qiu, and S. Yoshida, Practical quasi parton distribution functions, 1609.02018.

[7] J.-W. Chen, X. Ji, and J.-H. Zhang, Improved quasi parton distribution through Wilson line renormalization, 1609.08102 .

[8] G. S. Bali, B. Lang, B. U. Musch, and A. Schäfer, Novel quark smearing for hadrons with high momenta in lattice QCD, Phys. Rev. D93 (2016) 094515, [1602 . 05525].

[9] C. Alexandrou, K. Cichy, M. Constantinou, K. Hadjiyiannakou, K. Jansen, F. Steffens, et al., New Lattice Results for Parton Distributions, 1610.03689.

[10] R. Baron, P. Boucaud, J. Carbonell, A. Deuzeman, V. Drach, et al., Light hadrons from lattice QCD with light $(u, d)$, strange and charm dynamical quarks, JHEP 1006 (2010) 111, [1 004.5284 ].

[11] A. Hasenfratz and F. Knechtli, Flavor symmetry and the static potential with hypercubic blocking, Phys.Rev. D64 (2001) 034504, [hep-lat/ 010302 9].

[12] A. Martin, W. Stirling, R. Thorne, and G. Watt, Parton distributions for the LHC, Eur.Phys.J. C63 (2009) 189-285, [0901.0002].

[13] J. Owens, A. Accardi, and W. Melnitchouk, Global parton distributions with nuclear and finite- $Q^{2}$ corrections, Phys.Rev. D87 (2013) 094012, [1212 . 1702].

[14] S. Alekhin, J. Blümlein, and S. Moch, Parton Distribution Functions and Benchmark Cross Sections at NNLO, Phys.Rev. D86 (2012) 054009, [1202.2281].

[15] D. de Florian, R. Sassot, M. Stratmann, and W. Vogelsang, Extraction of Spin-Dependent Parton Densities and Their Uncertainties, Phys. Rev. D80 (2009) 034030, [0 904 . 3821].

[16] Jefferson Lab Angular Momentum collaboration, N. Sato, W. Melnitchouk, S. E. Kuhn, J. J. Ethier, and A. Accardi, Iterative Monte Carlo analysis of spin-dependent parton distributions, Phys. Rev. D93 (2016) 074005, [1601.07782]. 